\title{
The Optimal Controller Design of Buck-Boost Converter by using Adaptive Tabu Search Algorithm Based on State-Space Averaging Model
}

\author{
Jakkrit Pakdeeto*, Rangsan Chanpittayagit*, Kongpan Areerak ${ }^{\dagger}$ and Kongpol Areerak*
}

\begin{abstract}
Normally, the artificial intelligence algorithms are widely applied to the optimal controller design. Then, it is expected that the best output performance is achieved. Unfortunately, when resulting controller parameters are implemented by using the practical devices, the output performance cannot be the best as expected. Therefore, the paper presents the optimal controller design using the combination between the state-space averaging model and the adaptive Tabu search algorithm with the new criteria as two penalty conditions to handle the mentioned problem. The buck-boost converter regulated by the cascade PI controllers is used as the example power system. The results show that the output performance is better than those from the conventional design method for both input and load variations. Moreover, it is confirmed that the reported controllers can be implemented using the realistic devices without the limitation and the stable operation is also guaranteed. The results are also validated by the simulation using the topology model of MATLAB and also experimentally verified by the testing rig.
\end{abstract}

Keywords: Adaptive Tabu search, Buck-boost converter, State-space averaging model, Small-signal model, Controller design, Stability

\section{Introduction}

Presently, the artificial intelligence (AI) techniques are widely used for electrical engineering research areas such as the system identifications using adaptive tabu search (ATS) [1-5], the protection design in power system via ATS [6], the active power filter design using genetic algorithm (GA) [7], power loss minimization using particle swarm optimization (PSO) as well as artificial bee colony (ABC) [8], reactive power optimization for distribution systems based on ant colony optimization (ACO) [9], and etc. In this paper, the AI technique called the ATS method will be applied to design the cascade PI controllers of buck-boost converter because this algorithm has the mathematical proof to ensure that it can escape the local solutions [2]. However, when the AI is applied to the power electronic system, the main problem is the simulation time. This is because the simulation of power electronic system using software packages (such as MATLAB, PSIM, and etc.) provide a huge simulation time due to a switching behavior. It is not easily applicable for the AI searching method in which the iterative simulation is required. Moreover, many research works [10-13] normally present the optimal controller design using the AI methods in which the best output performance is guaranteed. Unfortunately, when

$\dagger$ Corresponding Author: Power electronics, Energy, Machines and Control Research Group, School of Electrical Engineering, Suranaree University of Technology, Thailand. (kongpan@sut.ac.th)

* Power electronics, Energy, Machines and Control Research Group, School of Electrical Engineering, Suranaree University of Technology, Thailand.

Received: June 28, 2016; Accepted: February 2, 2017 the resulting controllers are implemented, the output responses cannot provide the waveform with a good performance as expected. This is because the control signal is limited by the practical devices.

Therefore, to handle the mentioned problems, this paper also presents a state-space averaging model. The benefits of the proposed averaging model are concluded as follows:

- The fast computation time can be obtained via the averaging model. It is very useful for the optimal controller design using ATS algorithm in which the system responses are iteratively simulated.

- The control signal can be determined from the system state-variables. Hence, during the searching process, the control signal of each searched controller parameters can be identified. Consequently, the control signal limitation can be included as a penalty condition in the design process.

- The stability analysis can be also included by calculating the Eigenvalues from the small-signal model linearized from the state-space averaging model.

Hitherto, using the state-space averaging model in the searching process, the fast simulation time, the control signal consideration, and the stability confirmation can be achieved. As a result, the controller parameters designed by the proposed technique can provide the best output response that can be also implemented by practical devices. Furthermore, the stable operation is also confirmed. The three-phase uncontrolled rectifier feeding the regulated buck-boost converter is considered as the example system for this study. The ATS is applied to search the cascade PI 
controller parameters of buck-boost converter to achieve the best output voltage performance for both input and load variations. The proposed design procedure in the paper has not been reported in the previous publications. The simulation and experimental results for the system with the controller designed by the conventional method are compared with those of the system with the controller designed by the ATS method.

This paper is structured as follows. The considered power system is described in Section 2. The state-space averaging model derivation is explained in Section 3. In Section 4 , the optimal control design using the ATS method is illustrated in which the control signal consideration and the stability analysis are also included in the design process. Section 5 shows the controller design results validated by the simulation. Section 6 , the experimental results are presented to support the concept of the proposed design method. Finally, Section 7 concludes the advantages of the proposed design technique in which it is easily applicable to design the controller of other power converters.

\section{Considered Power System}

The considered power system is depicted in Fig. 1. It consists of balanced three-phase voltage source, transmission line parameters represented by $R_{e q}, L_{e q}$, and $C_{e q}, 6-$ pulse diode rectifier, DC-link filters represented by $R_{f}, L_{f}$, $R_{c}$, and $C_{f}$, buck-boost converter regulated by the cascade PI controllers feeding the resistive load represented by $R$. Generally, to regulate the $V_{o}$ output response, the cascade PI controllers having the inner-loop and outer-loop are used. The $K_{p i}$ and $K_{i i}$ are the PI controller parameters of inner-loop to control the inductor current of $L$, while the $K_{p v}$ and $K_{i v}$ are the PI controller parameters of outer-loop to control the output voltage of capacitor $C$. According to the example power system shown in Fig. 1, the aim of the paper is to design these PI controller parameters, here are
$K_{p i}, K_{i i}, K_{p v}$, and $K_{i v}$. These parameters are appeared in the proposed state-space averaging model. Therefore, the controllers based on their existing model can be tuned by using the ATS algorithm until the best output performance is achieved. Unfortunately, the regulated buck-boost converter in Fig. 1 normally behaves as a constant power load (CPL) in which it can significantly affect the system stability [14]. Hence, the stability study is also included in the tuning process via the small-signal model linearized from the state-space averaging model as the penalty condition. Moreover, dx in Fig. 1 is the control signal. This value is used to compare with the sawtooth waveform having amplitude equal to $A_{r}$. The $d_{x}$ can be calculated from the state-space averaging model. For the implementation, it should not be more than $A_{r}$. Therefore, this limitation is also included in the searching process as the penalty condition as well. It can be concluded that this paper presents the optimal controller design using the ATS algorithm to achieve the best output response. During the design process, two penalty conditions are included. The details how to design the controller using the proposed technique will be described in Section 4. The system parameters for the considered system are as follows: $V_{s}=$ $15 \mathrm{~V}_{\text {rms }} /$ phase, $f=50 \mathrm{~Hz}, R_{e q}=0.2 \Omega, L_{e q}=100 \mu \mathrm{H}, C_{e q}=$ $2 \mathrm{nF}, R_{f}=2 \Omega, L_{f}=33 \mathrm{mH}, C_{f}=1100 \mu \mathrm{F}, R_{c}=3 \Omega, L=15$ $\mathrm{mH}, C=1100 \mu \mathrm{F}$, and $R=80 \Omega$.

\section{State-Space Averaging Model}

It is well-known that the models of power converter are time-varying in nature because of their switching actions. In this paper, the DQ method [15-17] and the generalized state-space averaging (GSSA) method [18-20] are used to eliminate the switching behavior of diode and switch $Q$, respectively. As a result, the time-invariant model is achieved. Firstly, the DQ method is selected to derive the dynamic model of a three-phase diode rectifier in which

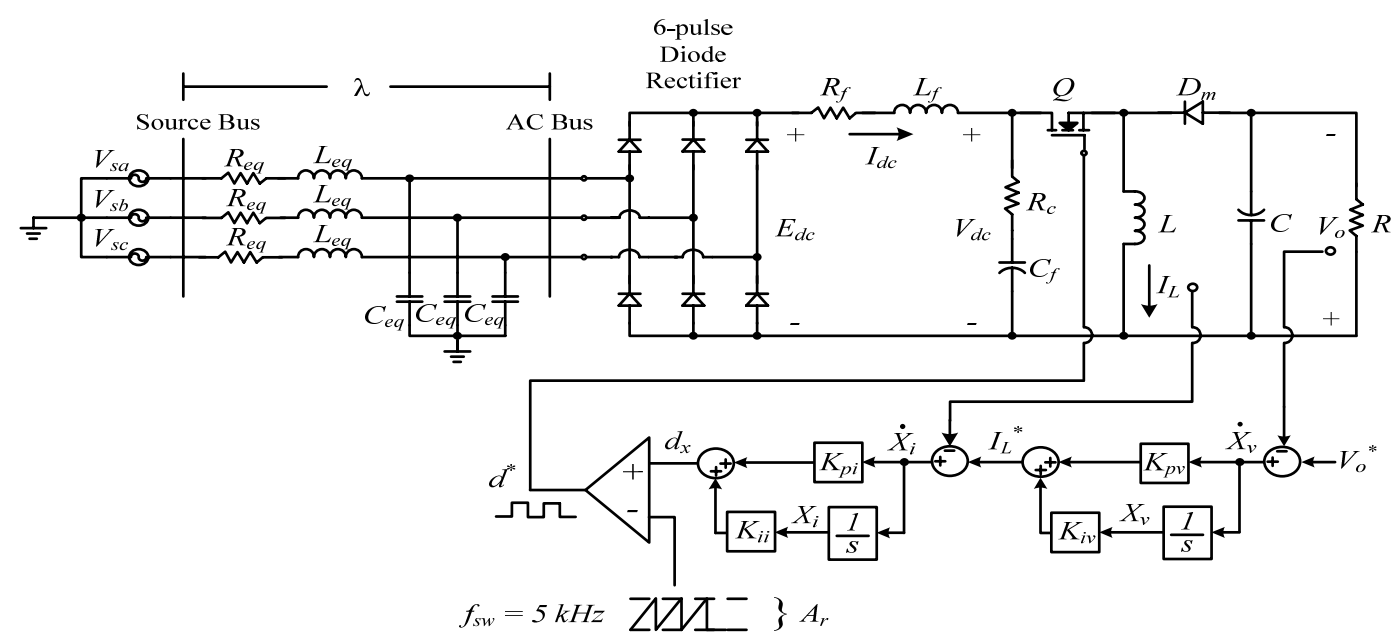

Fig. 1. Considered power system 


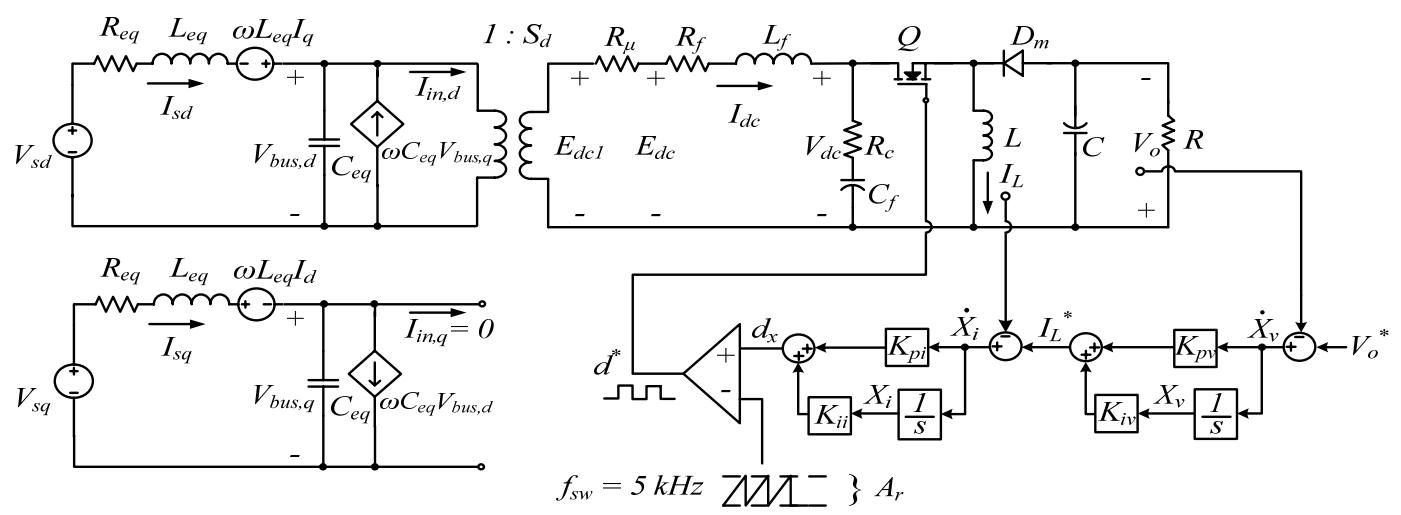

Fig. 2. The equivalent circuit of the considered system on DQ-axis

the diode rectifier can be treated as a transformer on DQaxis as shown in Fig. 2. According to Fig. 1, the effect of $L_{e q}$ on the AC side causes an overlap angle $\mu$ in the output waveforms that causes as a commutation voltage drop. This drop can be represented as a variable resistance $R_{\mu}$ that is located on the DC side as shown in Fig. 2. The transmission line section in Fig. 1 can also be transformed into DQ frame.

The DQ representation of the transmission line is then combined with the diode rectifier. As a result, the equivalent circuit of the considered power system can be represented in the DQ frame as depicted in Fig. 2 by fixing the rotating frame on the phase of the switching function $\left(\phi_{1}=\phi\right)$ [21]. In Fig. 2, the three-phase diode rectifier including the transmission line on AC side is already transformed into the DQ frame via the DQ method. Notice that the diode rectifier can be modeled as the transformer in which it can provide the time-invariant model. The GSSA modeling method is then used to eliminate the switching action (switch $Q$ ) of the buck-boost converter. The control signal $d_{x}$ can be calculated by the system state-variables given in (1). Notice that when PI controllers are considered, the $X_{v}$ of the voltage loop control and the $X_{i}$ of the current loop control are set as the state variables of the model.

$$
d_{x}=-K_{p i} I_{L}-K_{p v} K_{p i} V_{o}+K_{i v} K_{p i} X_{v}+K_{i i} X_{i}+K_{p v} K_{p i} V_{o}^{*}
$$

The control signal $d_{x}$ is compared with the sawtooth signal having the amplitude $A_{r}$ to provide the $d^{*}$ waveform. The $d^{*}$ is the duty cycle of buck-boost converter as well as the gate drive signal for the switch $Q$. Hence, $d^{*}$ should be equal to $0-1$ in which it can be calculated by (2).

$$
d^{*}=\frac{d_{x}}{A_{r}}
$$

Applying the basic KVL and KCL to Fig. 2 with the GSSA method and using (1) and (2) during the derivation process, the state-space averaging model of considered system can be expressed in (3).
The resulting model given in (3) is the nonlinear timeinvariant model in which it can be used to calculate the system responses with the fast computation time. It is very useful for the controller design using the ATS algorithm in which the output response is iteratively calculated. Furthermore, when the state-variables can be calculated by (3), the control signal $d_{x}$ can then be also determined by (1). As for the stability analysis, the model in (3) is linearized by using the first order terms of the Taylor expansion so as to achieve a set of linear differential equations around an equilibrium point. This resulting linearized model of (3) is then of the form in (4) that can be called small-signal model. Consequently, the eigenvalues can be determined from the Jacobian matrix $\mathbf{A}\left(\mathbf{x}_{\mathbf{o}}, \mathbf{u}_{\mathbf{o}}\right)$.

$$
\begin{aligned}
& \dot{I_{s d}}=-\frac{R_{e q}}{L_{e q}} I_{s d}+\omega I_{s q}-\frac{1}{L_{e q}} V_{b u s, d}+\frac{1}{L_{e q}} \sqrt{\frac{3}{2}} V_{m} \cos (\lambda) \\
& \dot{I_{s q}}=-\omega I_{s d}-\frac{R_{e q}}{L_{e q}} I_{s q}-\frac{1}{L_{e q}} V_{b u s, q}+\frac{1}{L_{e q}} \sqrt{\frac{3}{2}} V_{m} \sin (\lambda) \\
& \dot{V_{b u s, d}}=\frac{1}{C_{e q}} I_{s d}+\omega V_{b u s, q}-\sqrt{\frac{3}{2}} \cdot \frac{2 \sqrt{3}}{\pi C_{e q}} I_{d c} \\
& \stackrel{\bullet}{V_{b u s, q}}=\frac{1}{C_{e q}} I_{s q}-\omega V_{b u s, d} \\
& \dot{I_{d c}}=\sqrt{\frac{3}{2}} \cdot \frac{2 \sqrt{3}}{\pi L_{f}} V_{b u s, d}-\frac{\left(R_{\mu}+R_{f}+R_{c}\right)}{L_{f}} I_{d c}-\frac{1}{L_{f}} V_{d c}-\frac{R_{c} K_{p i}}{A_{r} L_{f}} I_{L}^{2} \\
& -\frac{R_{c} K_{p v} K_{p i}}{A_{r} L_{f}} I_{L} V_{o}+\frac{R_{c} K_{p i} K_{i v}}{A_{r} L_{f}} I_{L} X_{v}+\frac{R_{c} K_{i i}}{A_{r} L_{f}} I_{L} X_{i}+\frac{R_{c} K_{p v} K_{p i}}{A_{r} L_{f}} I_{L} V_{o}^{*} \\
& \dot{V_{d c}}=\frac{1}{C_{f}} I_{d c}+\frac{K_{p i}}{A_{r} C_{f}} I_{L}^{2}+\frac{K_{p v} K_{p i}}{A_{r} C_{f}} I_{L} V_{o}-\frac{K_{p i} K_{i v}}{A_{r} C_{f}} I_{L} X_{V}-\frac{K_{i i}}{A_{r} C_{f}} I_{L} X_{i}-\frac{K_{p v} K_{p i}}{A_{r} C_{f}} I_{L} V_{o}^{*} \\
& \dot{I_{L}}=-\frac{1}{L} V_{o}-\frac{K_{p i}}{A_{r} L} V_{d c} I_{L}-\frac{K_{p v} K_{p i}}{A_{r} L} V_{d c} V_{o}+\frac{K_{p i} K_{i v}}{A_{r} L} V_{d c} X_{v}+\frac{K_{i i}}{A_{r} L} V_{d c} X_{i}+\frac{K_{p v} K_{p i}}{A_{r} L} V_{d c} V_{o}^{*} \\
& -\frac{K_{p i}}{A_{r} L} V_{o} I_{L}-\frac{K_{p v} K_{p i}}{A_{r} L} V_{o}^{2}+\frac{K_{p i} K_{i v}}{A_{r} L} V_{o} X_{v}+\frac{K_{i i}}{A_{r} L} V_{o} X_{i}+\frac{K_{p v} K_{p i}}{A_{r} L} V_{o} V_{o}^{*} \\
& \dot{V}_{o}=\frac{1}{C} I_{L}+\frac{K_{p i}}{A_{r} C} I_{L}^{2}+\frac{K_{p v} K_{p i}}{A_{r} C} I_{L} V_{o}-\frac{K_{p i} K_{i v}}{A_{r} C} I_{L} X_{v}-\frac{K_{i i}}{A_{r} C} I_{L} X_{i}-\frac{K_{p v} K_{p i}}{A_{r} C} I_{L} V_{o}^{*}-\frac{1}{R C} V_{o} \\
& \dot{X}_{v}=-V_{o}+V_{o}^{*} \\
& \dot{X}_{i}=-I_{L}-K_{p v} V_{o}+K_{i v} X_{v}+K_{p v} V_{o}^{*}
\end{aligned}
$$

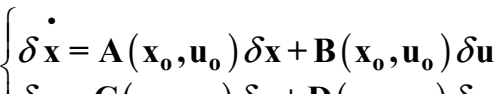


where

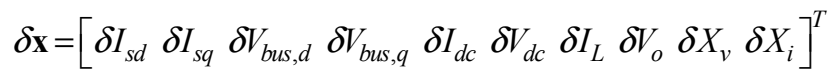

$$
\begin{aligned}
& \delta \mathbf{u}=\left[\begin{array}{ll}
\delta V_{s} & \delta V_{o}^{*}
\end{array}\right]^{T} \\
& \delta \mathbf{y}=\left[\begin{array}{ll}
\delta V_{d c} & \delta V_{o}
\end{array}\right]^{T} \\
& \mathbf{B}=[]_{10 \times 2} \quad \mathbf{C}=[]_{2 \times 10} \mathbf{D}=[]_{2 \times 2}
\end{aligned}
$$

The matrices A B C and D are too awkward to put in this paper. The more details how to derive the considered power system using the DQ and GSSA methods can be found in [21].

\section{Optimal Controller Design}

The ATS is one of the powerful artificial intelligence (AI) search algorithms and has a convergence property [2]. Therefore, in this paper, the ATS algorithm is used as the tuning mechanism to minimize $J$ until one of the termination criteria is satisfied. The use of ATS algorithm to design the cascade PI controller via the state-space averaging model is explained in the block diagram of Fig. 3 in which two penalty conditions are included in the process. Referring to Fig. 3, the command input $V_{o}^{*}$ is firstly determined. Then, all state $x_{i}$ values can be calculated from the proposed state-space averaging model given in (3). In terms of computational time, if the system of Fig. 1 is simulated from $t=0-1.5 \mathrm{~s}$. by using the exact topological model in MATALB, the computing time is equal to $\sim 175 \mathrm{~s}$. Otherwise, the system response under the same scenario calculated from the proposed dynamic model given in (3) consumes only $\sim 0.08 \mathrm{~s}$. Hence, for the searching case, the system will be repeatedly simulated more than 100 rounds. Applying the obtained models for simulations instead of the exact topology models based on the simulation packages is appropriate. As can be seen in Fig. 3, there are three parts in the tuning design process as follows:

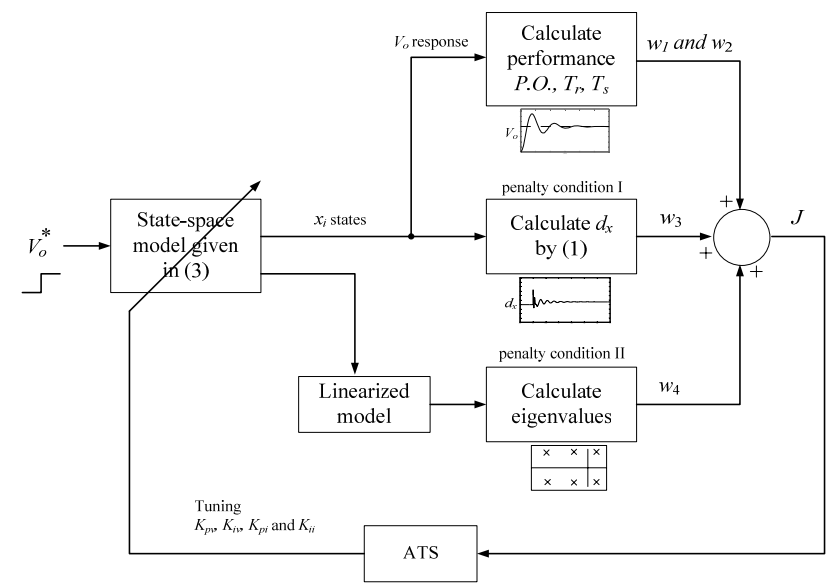

Fig. 3. ATS-based cascade PI controller optimization

\subsection{Calculating the performance index}

The output voltage response $V_{o}$ (one of state-variables) is used for this section. The dynamic response of $V_{o}$ for both varying command voltage and load conditions can be obtained. In the paper, the changing $V_{o}^{*}$ from $20 \mathrm{~V}$ to $30 \mathrm{~V}$ with $R=80 \Omega$ and changing $R$ from $80 \Omega$ to $40 \Omega$ with $V_{o}^{*}=30 \mathrm{~V}$ are used for the design process. As a result, the percent overshoot $\left(P . O_{A T S}\right)$, rise time $\left(T_{r, A T S}\right)$, and setting time $\left(T_{s, A T S}\right)$ are determined. The weighting function $w_{l}$ (for input voltage variation) and $w_{2}$ (for load variation) can be calculated by (5)

$$
w_{1} \text { and } w_{2}=h_{1}\left(\frac{P . O \cdot A T S}{P . O_{C O N}}\right)+h_{2}\left(\frac{T_{r, A T S}}{T_{r, C O N}}\right)+h_{3}\left(\frac{T_{s, A T S}}{T_{s, C O N}}\right)
$$

where $P . O_{C O N}, T_{r, C O N}$, and $T_{s, C O N}$ are the percent overshoot, rise time, and setting time of $V_{o}$ response when the controllers are designed by the conventional method. The conventional design method is based on the standard second-order system characteristic. More details how to design the controllers using the conventional method can be found in Appendix. In (5), the $h_{1}, h_{2}$, and $h_{3}$ are the priority coefficients of P.O., $T_{r}$, and $T_{s}$ in which the summation of these values must be equal to 1 . In this paper, the values of $h_{1}, h_{2}$, and $h_{3}$ are set to $0.34,0.33$, and 0.33 , respectively because the P.O., $T_{r}$, and $T_{s}$ are equally significant. Note that, the $w_{1}$ and $w_{2}$ becomes to 1 for the conventional design method.

\subsection{Penalty condition I}

The control signal consideration is included in the design process to ensure that the resulting PI controller parameters can be implemented by using the realistic devices. The control signal $d_{x}$ can be calculated from the system state values by (1). The $w_{3}$ can be determined by using the condition given in (6) in which the $A_{r}$ is the amplitude of sawtooth signal. In the paper, $A_{r}$ is set equal to $10 \mathrm{~V}$. The $A_{r}$ cannot be set exceed $15 \mathrm{~V}$ if the controllers are implemented by using op-amp as the comparator because it will be saturated at the voltage level equal to $\sim 15 \mathrm{~V}$.

$$
\begin{aligned}
& \text { if } d_{x} \leq A_{r} \\
& w_{3}=0 \\
& \text { else } \\
& w_{3}=10 \\
& \text { end }
\end{aligned}
$$

\subsection{Penalty condition II}

As mentioned before, the regulated buck-boost converter behaves as a CPL in which this load can significantly degrade the system stability. Hence, during tuning PI controller parameters, the stability analysis is also included. 
According to Fig. 3, the state-space model is linearized by the first-order term of Taylor series expansion. Consequently, all eigenvalues $\lambda_{i}$ can be calculated from the matrix A of linearized model or small-signal model. The $w_{4}$ can be determined by using the condition given in (7).

$$
\begin{aligned}
& \text { if } \operatorname{Re}\left\{\lambda_{i}\right\}<0 \\
& w_{4}=0 \\
& \text { else } \\
& w_{4}=10 \\
& \text { end }
\end{aligned}
$$

All $w_{1} w_{2} w_{3}$ and $w_{4}$ can be calculated from the proposed state-space averaging model given in (3) in which the PI controller parameters $K_{p v} K_{i v} K_{p i}$ and $K_{i i}$ are appeared inside the model. The ATS algorithm will tune these controller parameters until the minimum $J$ is achieved. In the paper, the objective function $J$ is calculated by (8) in which $y$ is equal to 4 because there are only 4 conditions for the design procedure. The designer can add other conditions for different considerations.

$$
J=\sum_{x=1}^{y} w_{x}
$$

The number of iterative is set to 100 as the termination criteria. The upper and lower limits of $K_{p v} K_{i v} K_{p i}$ and $K_{i i}$ are set to [0.015 0.4], [1.4 30], [0.4 10], and [500 10000], respectively. The setting boundary of these parameters can be randomly determined. However, for this paper, this setting boundary is based on the controller designed by using the conventional method. As a result, the optimal PI controller can be achieved with a fast computing time. Notice that the penalty values of $w_{3}$ and $w_{4}$ are set equal to 10. However, some problems can be set to different values depending on the $w_{1}$ and $w_{2}$ value. In this paper, the penalty values equal to 10 is sufficient to evaluate the bad solution because the minimum value of $J$ is around 0.5 . In addition, the steady-state error is not considered in the design process of Fig. 3. This is because the PI controllers increase the system type. Therefore, the steady-state error is always zero.

\section{Simulation Results}

The resulting PI controller parameters with their objective function values represented by $J$ are given in Table 1.

According to Table 1, the ATS method can provide the minimum $J$ compared with the conventional method. It means that the best output voltage performance can be achieved by using the ATS algorithm technique. Moreover, the implementation ability and the stable operation are guaranteed. To verify the results, Fig. 4(a) shows the $V_{o}$
Table 1. The comparison results between conventional method and ATS method

\begin{tabular}{c|c|c}
\hline PI controller parameters/ & \multicolumn{2}{|c}{ Design method } \\
\cline { 2 - 3 }$J_{\text {value }}$ & Conventional method & ATS method \\
\hline$K_{p i}$ & 5.486 & 1.457 \\
\hline$K_{i i}$ & 2742 & 4.573 \\
\hline$K_{p v}$ & 0.128 & 0.5453 \\
\hline$K_{i v}$ & 7.04 & 22.332 \\
\hline$J$ & 1.0 & 0.377 \\
\hline
\end{tabular}
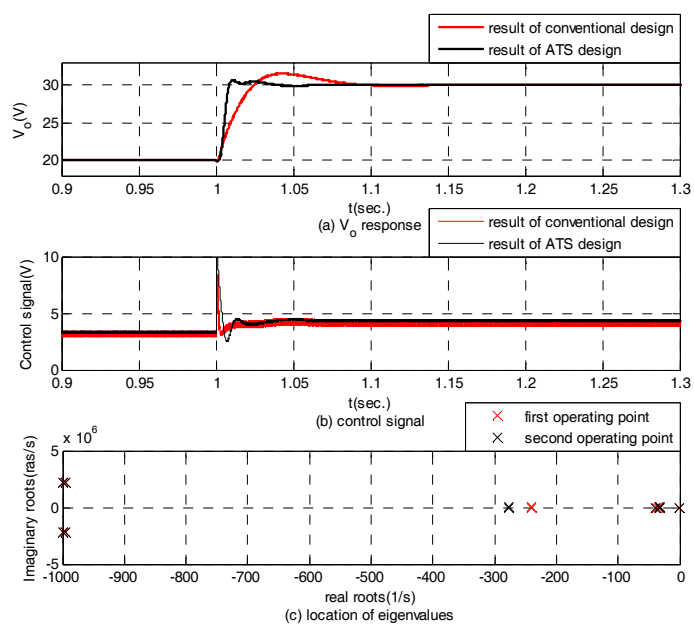

Fig. 4. $V_{o}^{*}$ changing from $20 \mathrm{~V}$ to $30 \mathrm{~V}$

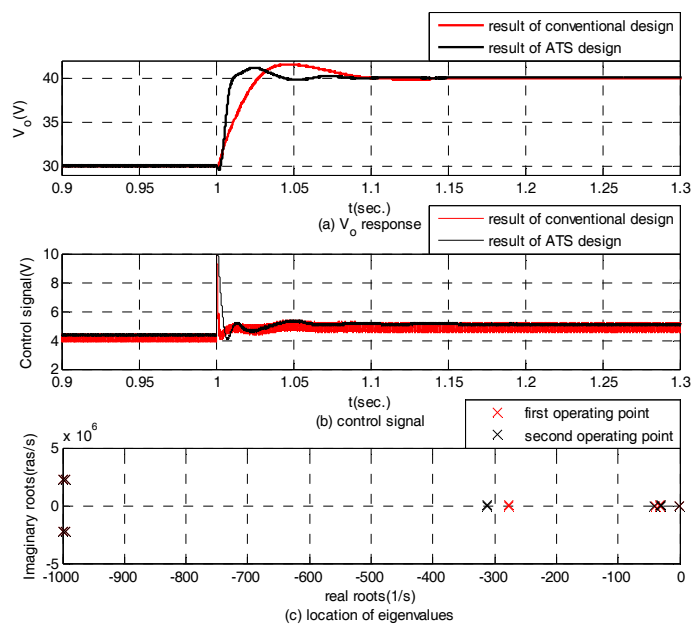

Fig. 5. $V_{o}^{*}$ changing from $30 \mathrm{~V}$ to $40 \mathrm{~V}$

response to a step change of $V_{o}^{*}$ from 20 to $30 \mathrm{~V}$ that occurs at $t=1 \mathrm{~s}$. It can be seen from the simulation result that the cascade PI controller designed from the ATS method can provide the better output performance than those designed from the conventional method. For this case, the control signal $d_{x}$ is shown in Fig. 4(b). As can be seen in this figure, the resulting control signal is not exceeded $A_{r}$ value $(10 \mathrm{~V})$. Hence, the PI controller parameters designed by the ATS method can be implemented. For example, if this control signal is compared with sawtooth having $A_{r}=$ 


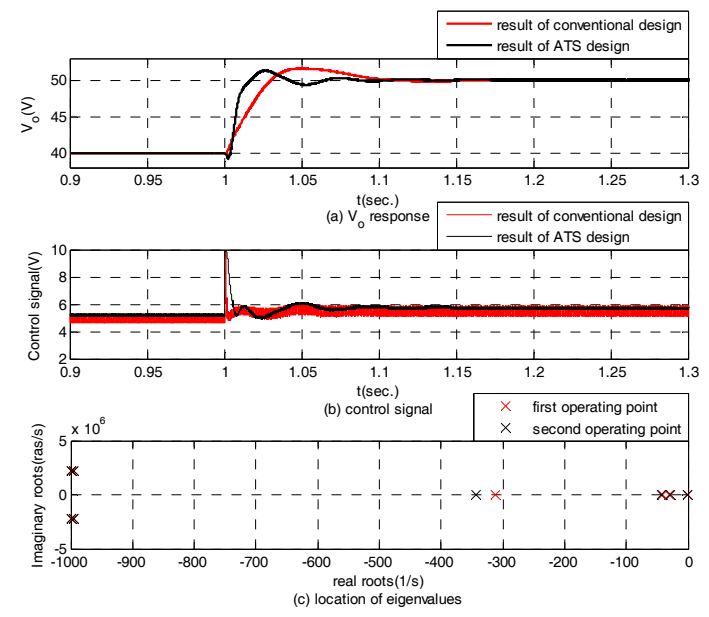

Fig. 6. $V_{o}^{*}$ changing from $40 \mathrm{~V}$ to $50 \mathrm{~V}$
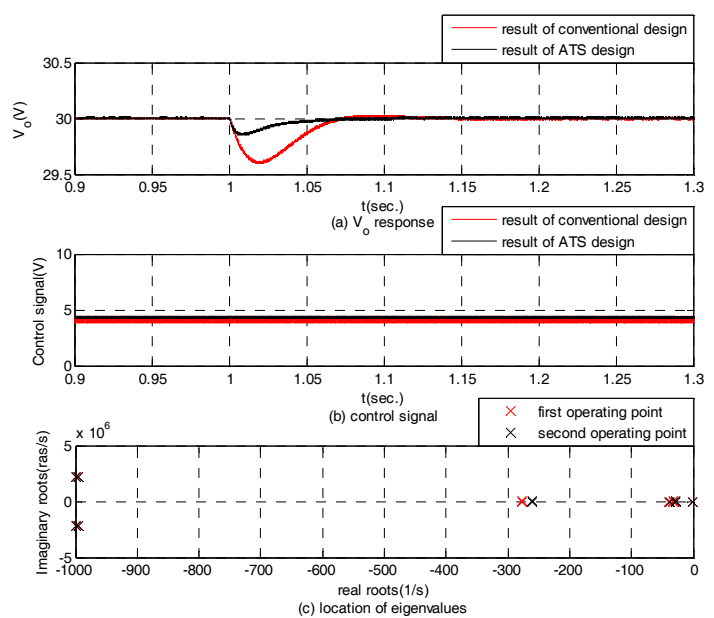

Fig. 7. $V_{o}^{*}=30 \mathrm{~V}$ with $\mathrm{R}$ changed from $80 \Omega$ to $40 \Omega$

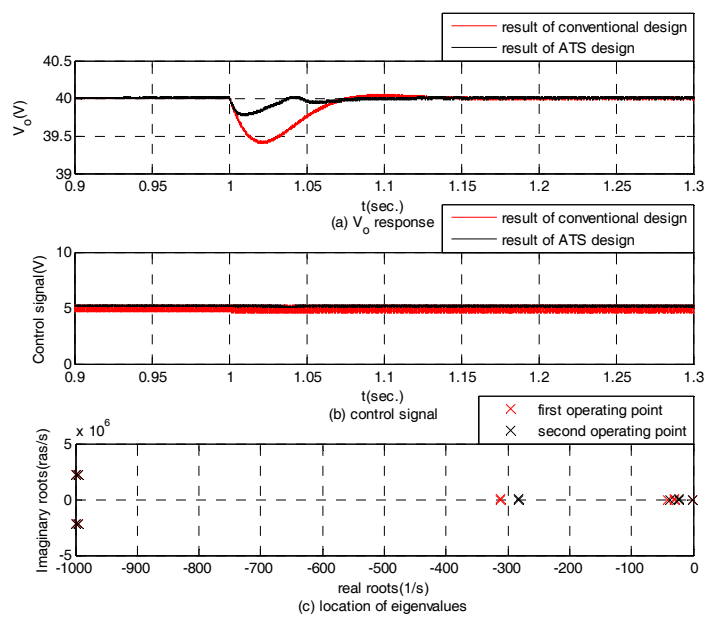

Fig. 8. $V_{o}^{*}=40 \mathrm{~V}$ with $\mathrm{R}$ changed from $80 \Omega$ to $40 \Omega$

10. The op-amp will be not saturated. In terms of stability analysis, the eigenvalues are addressed in Fig. 4(c) in which all eigenvalues are located on the stable region. For varying voltage command from $30 \mathrm{~V}$ to $40 \mathrm{~V}$ and $40 \mathrm{~V}$

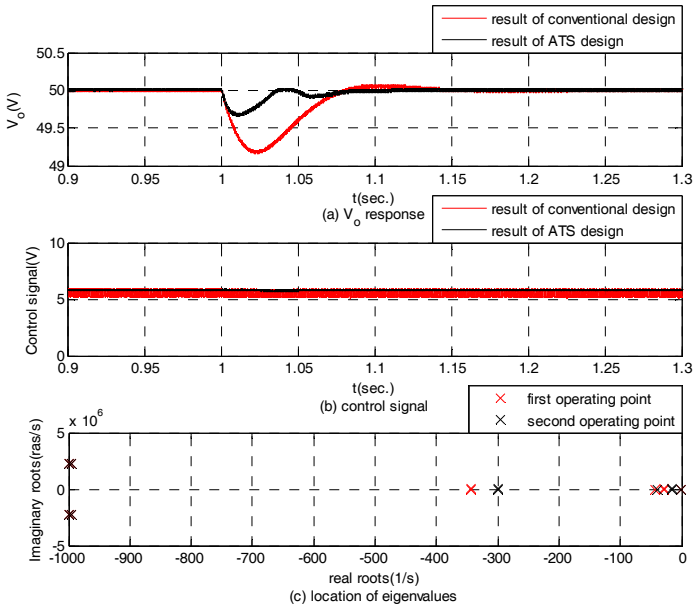

Fig. 9. $V_{o}^{*}=50 \mathrm{~V}$ with $\mathrm{R}$ changed from $80 \Omega$ to $40 \Omega$

to $50 \mathrm{~V}$, the results of $V_{o}$ response, control signal, and location of eigenvalues are depicted in Fig. 5 and Fig. 6, respectively.

For varying load conditions, Fig. 7(a) shows the $V_{o}$ response when $V_{o}^{*}$ is set to $30 \mathrm{~V}$ and resistive load $\mathrm{R}$ is changed from $80 \Omega$ to $40 \Omega$. The control signal and the result of stability analysis for this case are also presented in Fig. 7(b) and Fig. 7(c), respectively. Under the same condition of varying load, the results for $V_{o}^{*}$ equal to $40 \mathrm{~V}$ and $50 \mathrm{~V}$ are shown in Fig. 8 and Fig. 9, respectively.

The simulation results from Fig. 4 - Fig. 9 confirm that the ATS method can provide the better output voltage performance compared with the conventional method. Although changing $V_{o}^{*}$ from $20 \mathrm{~V}$ to $30 \mathrm{~V}$ with $R=80 \Omega$ and changing $R$ from $80 \Omega$ to $40 \Omega$ with $V_{o}^{*}=30 \mathrm{~V}$ were only used in the ATS process, the better output voltage performance can be obtained for other conditions.

The system with the PI controllers designed by the ATS method still provide the better output voltage response compared with those of conventional method.

\section{Experimental Results}

It has been established in the previous section that the proposed ATS design can provide the better output voltage response than those from the conventional design for both input and load variations. In this section, the experimental validation is presented. The experimental rig is shown in Fig. 10. The cascade PI controllers based on the diagrammatic representation of Fig. 1 were implemented using an Atmaga1280 microcontroller that highlighted by the number 3 in Fig. 10. The resulting PI controller parameters were applied to the system of Fig.10 to regulate the output voltage. The simulation and experimental rig were subjected to the same condition. The resulting $V_{o}$ waveforms by using PI controller parameters designed from both conventional and ATS methods for changing 


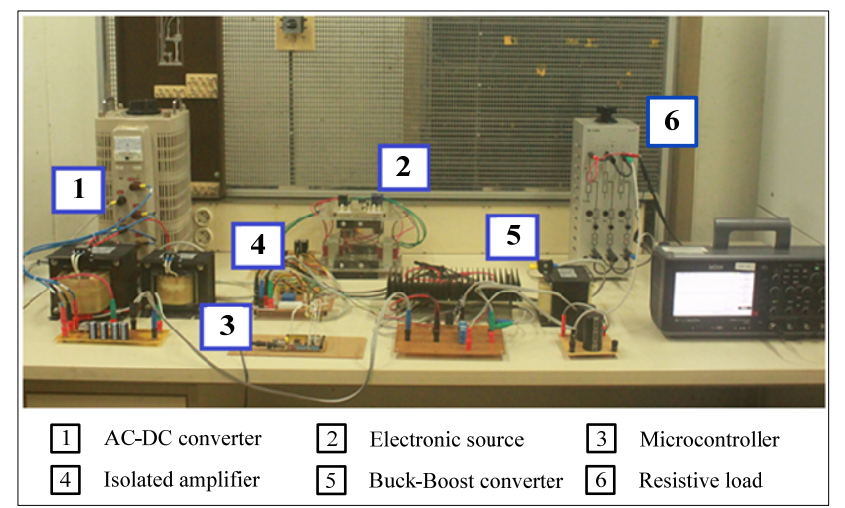

Fig. 10. The testing rig of the considered power system

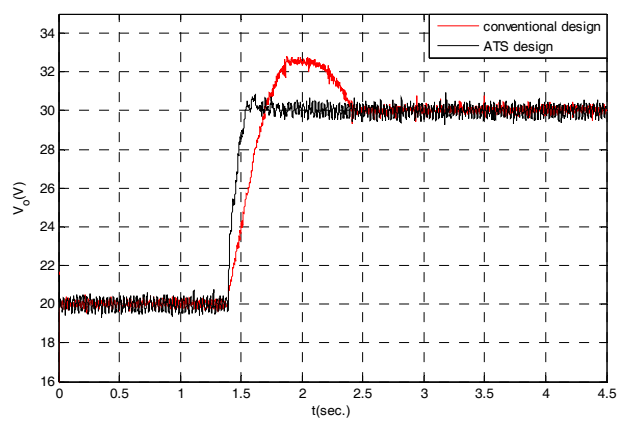

Fig. 11. The experimental results for changing $V_{o}^{*}$ from $20 \mathrm{~V}$ to $30 \mathrm{~V}$

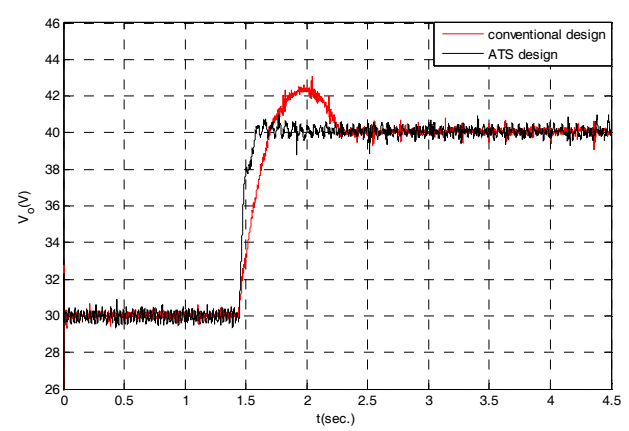

Fig. 12. The experimental results for changing $V_{o}^{*}$ from $30 \mathrm{~V}$ to $40 \mathrm{~V}$

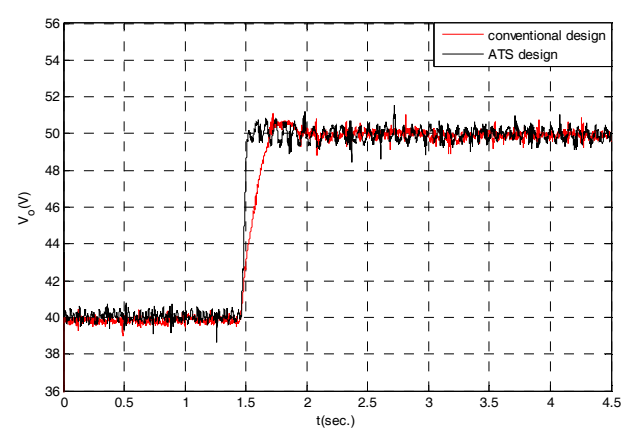

Fig. 13. The experimental results for changing $V_{o}^{*}$ from $40 \mathrm{~V}$ to $50 \mathrm{~V}$

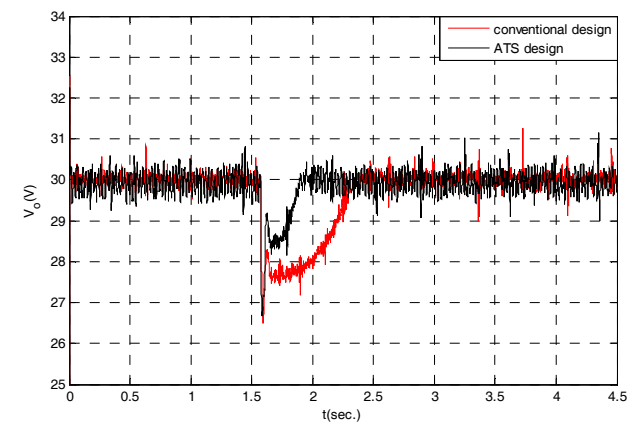

Fig. 14. The experimental results for changing $R$ from $80 \Omega$ to $40 \Omega$ with $V_{o}^{*}=30 \mathrm{~V}$

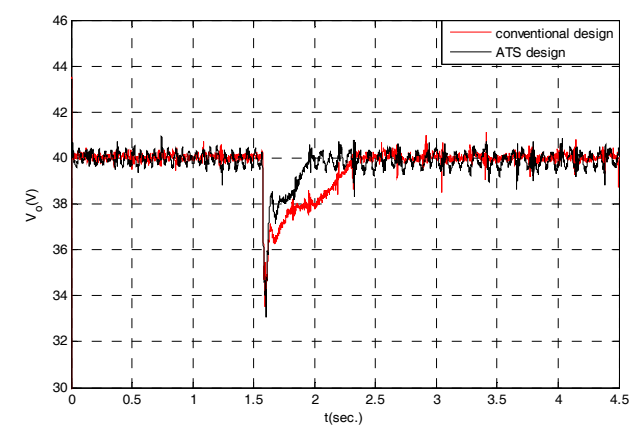

Fig. 15. The experimental results for changing $R$ from $80 \Omega$ to $40 \Omega$ with $V_{o}^{*}=40 \mathrm{~V}$

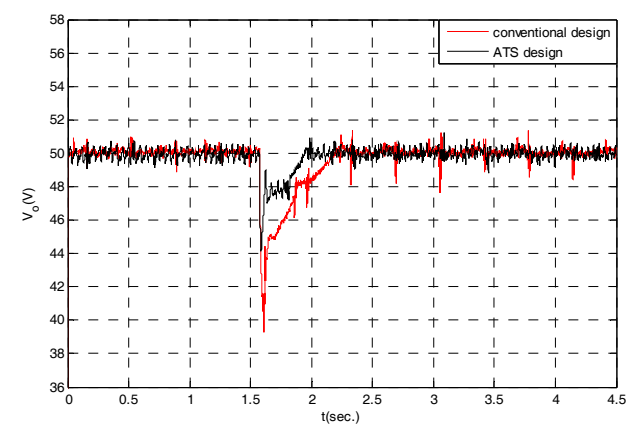

Fig. 16. The experimental results for changing $R$ from $80 \Omega$ to $40 \Omega$ with $V_{o}^{*}=50 \mathrm{~V}$

$V_{o}^{*}$ from $20 \mathrm{~V}$ to $30 \mathrm{~V}, 30 \mathrm{~V}$ to $40 \mathrm{~V}$, and $40 \mathrm{~V}$ to $50 \mathrm{~V}$ with $R=80 \Omega$ are shown in Figs. 11 - Fig. 13, respectively. The comparison waveforms for load variations when resistive load $R$ is changed from $80 \Omega$ to $40 \Omega$ with $V_{o}^{*}$ equal to $30 \mathrm{~V}, 40 \mathrm{~V}$, and $50 \mathrm{~V}$ are shown in Figs. 14-Fig. 16, respectively.

Overall it can be concluded that a good agreement between simulation and experiment results during the input and load variation can be achieved. The benefit when the system using the controllers designed by the proposed ATS method is clearly shown. 


\section{Conclusion}

According to the results, it indicates that the state-space averaging model and the ATS algorithm play a significant mechanism to the optimal controller design process. The ATS algorithm is very useful to design the cascade PI controllers of buck-boost converter. Because of the penalty condition I and II, the resulting controller parameters are confirmed that they can be implemented using the practical devices without the saturated problems and the stable operation can be achieved. During the tuning process, the control signal $d_{x}$ and the eigenvalues can be calculated from the proposed averaging model. The results have been already verified by using the simulation and experiment with a good agreement. The design procedure proposed in the paper is not limited to only buck-boost converter, but it can be also applied to all power converters with various types of controllers. However, the averaging models of the system are very important in which they must be determined before using the proposed design process. Furthermore, other AI algorithms such as GA, PSO, and etc. can be applied to the design process using the same procedure as well. It can be seen that the proposed design technique is very flexible and simple for the optimal controller design of power converter in which the implementation ability and the stable operation are confirmed.

\section{Appendix}

The details of classical method for PI controller design are as follow:

\section{- Current loop control}

The schematic of the current loop control of the system in Fig. 1 is shown in Fig. A1.

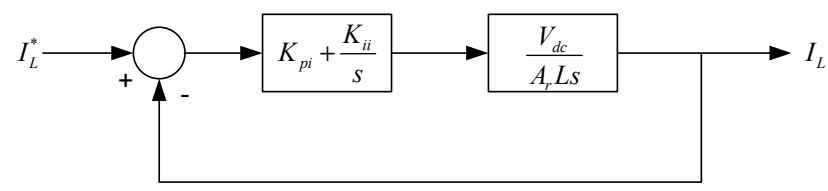

Fig. A1. current loop control

In Fig. A1, the $K_{p i}$ and $K_{i i}$ are the PI parameters of current loop control, while $L, A_{r}$, and $V_{d c}$ are the inductor of buck-boost converter, amplitude of sawtooth waveform, and DC-link voltage, respectively. Closed-loop transfer function of the current loop is given by:

$$
\frac{I_{L}}{I_{L}{ }^{*}}=\frac{\left(\frac{K_{p i} s+K_{i i}}{A_{r} L}\right) V_{d c}}{s^{2}+\left(\frac{K_{p i} V_{d c}}{A_{r} L}\right) s+\frac{K_{i i} V_{d c}}{A_{r} L}}
$$

The closed-loop denominator has roots with $\omega_{n i}$ and $\zeta_{i}$. The standard second order form is

$$
s^{2}+2 \zeta \omega_{n} s+\omega_{n}^{2}
$$

Hence, the current loop controller can be designed by comparing between the denominator of (A-1) and (A-2) to yield:

$$
\begin{gathered}
K_{p i}=\frac{2 \zeta_{i} \omega_{n i} A_{r} L}{V_{d c}} \\
K_{i i}=\frac{\omega_{n i}^{2} A_{r} L}{V_{d c}}
\end{gathered}
$$

\section{- Voltage loop control}

The schematic of the voltage loop control of the system in Figure1 is shown in Fig. A2.

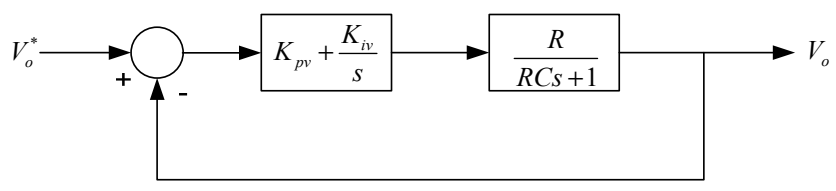

Fig. A2. voltage loop control

In Fig. A2, the $K_{p v}$ and $K_{i v}$ are the PI parameters of voltage loop control, while $C$ and $R$ are the capacitor of buck-boost converter and resistive load, respectively. Closed-loop transfer function of the voltage loop is given by:

$$
\frac{V_{o}}{V_{o}^{*}}=\frac{\left(\frac{K_{p v} s+K_{i v}}{C}\right)}{s^{2}+\left(\frac{K_{p v} R+1}{R C}\right) s+\frac{K_{i v}}{C}}
$$

Therefore, the voltage loop controller can be also designed by comparing between the denominator of (A-5) and (A-2) to yield:

$$
\begin{gathered}
K_{p v}=\frac{2 \zeta_{v} \omega_{n v} R C-1}{R} \\
K_{i v}=\omega_{n v}^{2} C
\end{gathered}
$$

In this paper, the PI controllers of both current and voltage control loops are designed by using (A-3), (A-4), $(\mathrm{A}-6)$, and $(\mathrm{A}-7)$. It can be seen that the controllers depend on the system parameters, damping ratio $\zeta_{i}$ and $\zeta_{v}$, the bandwidths of current loop $\omega_{n i}$, and voltage loop $\omega_{n v}$. The PI parameters in Table 1 of Section 5 (conventional method) are designed by selecting $\zeta_{v}=0.8, \zeta_{i}=0.8, \omega_{n i}=800$ $\mathrm{rad} / \mathrm{s}, \omega_{n v}=80 \mathrm{rad} / \mathrm{s}$, and $V_{d c}=35 \mathrm{~V}$. 


\section{Acknowledgements}

This work was supported by Suranaree University of Technology (SUT).

\section{References}

[1] D. Puangdownreong, K-N. Areerak, A. Srikaew, S. Sujijorn and P. Totarong, "System Identification via Adative Tabu Search," In Proceedings IEEE International Conference on Industrial Technology (ICIT02), 2002, pp. 915-920.

[2] S. Sujitjorn, T. Kulworawanichpong and D. Puangdownreong and K-N Areerak, "Adaptive Tabu Search and Applications in Engineering Design," Book Chapters in Integrated Intelligent Systems for Engineering Design (ed. X. F. Zha and R.J. Howlett), IOS Press, The Netherlands, 2006, pp. 233-257.

[3] D. Puangdownreong, K-N. Areerak, K-L. Areerak, T. Kulworawanichpong, and S. Sujitjorn, "Application of adaptive tabu search to system identification," IASTED International Conference on Modelling, Identification, and Control (MIC2005), Innsbruck, Austria: February 16-18, 2005, pp. 178-183.

[4] T. Kulworawanichpong, K-L. Areerak, K-N. Areerak, P. Pao-la-or, D. Puangdownreong, and S. Sujitjorn, "Dynamic parameter identification of induction motors using intelligent search techniques," IASTED International Conference on Modelling, Identification, and Control (MIC2005), Innsbruck, Austria: February 16-18, 2005, pp. 328-332.

[5] T. Kulworawanichpong, K-L. Areerak, K-N. Areerak, and S. Sujitjorn, "Harmonic Identification for Active Power Filters Via Adaptive Tabu Search Method," LNCS(Lecture Notes in Computer Science), SpringerVerlag Heidelberg, vol. 3215, 2004, pp. 687-694.

[6] K.-N. Areerak, T. Kulworawanichpong and S. Sujitjorn, "Moving Towards a New Era of Intelligent Protection through Digital Relaying in Power Systems," Lecture Notes in Computer Science, SpringerVerlag Heidelberg, vol. 3213, 2004, pp. 1255-1261.

[7] T. Narongrit, K-L. Areerak, and K-N. Areerak, "Design of an Active Power Filter using Genetic Algorithm Technique," The $9^{\text {th }}$ WSEAS International Conference on Artificial Intelligent, Knowledge Engineering and Data Bases (AIKED'10), Cambridge, United Kingdom: February 20-22, 2010, pp. 46-50.

[8] U. Leeton, D. Uthitsunthorn, U. Kwannetr, N. Sinsuphun, T. Kulworawanichpong, "Power loss minimization using optimal power flow based on particle swarm optimization," International Conference on Electrical Engineering / Electronics Computer Telecommunications and Information Technology (ECTI-CON), Chaing Mai: May 19-21, 2010, pp. 440-444.
[9] G. Lirui, H. Limin, Z. Liguo, L. Weina, and H. Jie, "Reactive Power Optimization for sidtribution systems based on Dual Population Ant Colony Optimization," in Proc. $27^{\text {th }}$ Chinese Control Conference (CCC 2008)., China, pp. 89-93.

[10] B. Achiammal, R. Kayalvizhi, "Genetic Algorithm based PI controller for Negative Output Elementary LUO converter," Advanced Communication Control and Computing Technologies (ICACCCT), 2014 International Conference., 2014, pp. 1099-1103.

[11] H. Madadi Kojabadi, Q. Cao, L. Chang, M. Ghribi, A. Dupuis, "Optimal PI controller gains using a multiloop multi-objective genetic algorithm in IM drives," Electrical and Computer Engineering, 2005. Canadian Conference, pp. 470-473.

[12] Xutao Li, Minjie Chen, Y. Tsutomu, "A method of searching PID controller's optimized coefficients for Buck converter using particle swarm optimization," Power Electronics and Drive Systems (PEDS), 2013 IEEE 10th International Conference. pp. 238-243.

[13] E. Sahin, M.S. Ayas, I.H. Altas, "A PSO optimized fractional-order PID controller for a PV system with DC-DC boost converter," Power Electronics and Motion Control Conference and Exposition (PEMC), 2014 16th International, pp. 477-481.

[14] C. Rivetta, G.A. Williamson, and A. Emadi, "Constant Power Loads and Negative Impedance Instability in Sea and Undersea Vehicles Statement of the Problem and Comprehensive Large-Signal Solution," in Proc. IEEE Electric Ship Tech. Symposium., Philadelphia, PA USA, July 2005, pp. 313-320.

[15] C.T. Rim, D.Y. Hu, and G.H. Cho, "Transformers as Equivalent Circuits for Switches: General Proof and D-Q Transformation-Based Analysis," IEEE trans. On Indus. Appl., vol. 26, no. 4, July/August 1990, pp. 777-785.

[16] K.-N. Areerak, S.V. Bozhko, G.M. Asher, and D.W.P. Thomas, "DQ-Transformation Approach for Modeling and Stability Analysis of AC-DC Power System with Controlled PWM Rectifier and Constant Power Load," $13^{\text {th }}$ International Power Electronics and Motion Control Conference (EPE-PEMC 2008), Poznan, Poland, 1-3 September 2008.

[17] K. Chaijarurnudomrung, K-N. Areerak, and K-L. Areerak, "Modeling of Three-phase Controlled Rectifier using a DQ method," 2010 International Conference on Advances in Energy Engineering (ICAEE 2010), Beijing, China: June 19-20, 2010, pp. 56-59.

[18] J. Mahdavi, A. Emadi, M.D. Bellar, M. Ehsani, "Analysis of Power Electronic Converter Using the Generalized State-Space Averaging Approach," IEEE Trans. On Circuit and System., vol. 44, August 1997, pp. 767-770.

[19] A. Emadi, "Modeling and Analysis of Multiconverter DC Power Electronic System Using the Generalized 
State-Space Averaging Method," IEEE Trans. On Indus. Elect., vol. 51, n. 3, June 2004, pp. 661-668.

[20] Ngamkong P., Kochcha P., Areerak K-N, Sujitjorn S. and Areerak K-L., "Application of the generalized state-space averaging method to modeling of DC-DC Power Converters," Mathematical and Computer Modelling of Dynamical Systems, vol. 18, no. 3, June 2012, pp. 243-260.

[21] Rangsan Chanpittayagit, Kongpan Areerak, and Kongpol Areerak (2014). "Modeling of AC-DC Power System Feeding a Controlled Buck-Boost Converter," Electrical Engineering/Electronics, Computer, Telecommunications and Information Technology (ECTI-CON), 2014 11th International Conference. pp. 1-6.

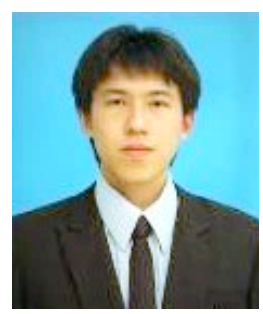

Jakkrit Pakdeeto was born in Nakhon Ratchasima, Thailand, in 1991. He received the B.Eng. (first-class honors) and M.Eng. degrees in electrical engineeering from Suranaree University of Technology (SUT), Nakhon Ratchasima, Thailand, in 2013 and 2015, respectively where he is currently studying the Ph.D. degree in electrical engineering. His main research interests include stability analysis, power electronics, AI applications, control systems and DC micro-grid systems.

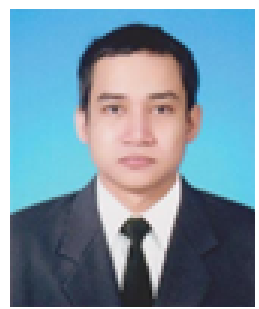

Rangsan Chanpittayagit received the B.Eng. and M.Eng degrees in electrical engineering from Suranaree University of Technology (SUT), Nakhon Ratchasima, Thailand, in 2012 and 2015, respectively. His main research interests include stability analysis, power electronics, AI applications, and control systems.

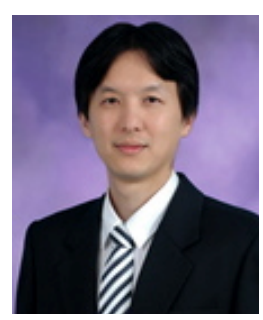

Kongpol Areerak received the B.Eng, M.Eng, and Ph.D. degrees in electrical engineering from Suranaree University of Technology (SUT), Thailand, in 2000, 2003, and 2007, respectively. Since 2007, he has been a lecturer and Head of Power Quality Research Unit (PQRU) in the School of Electrical Engineering, SUT. He received the Associate Professor in Electrical Engineering in 2015. His main research interests include active power filter, harmonic elimination, artificial intelligence applications, motor drive, and intelligence control systems.

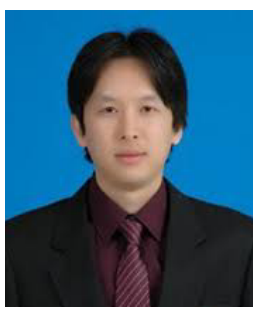

Kongpan Areerak received the B.Eng. M.Eng degrees from Suranaree University of Technology (SUT), Nakhon Ratchasima, Thailand, in 2000 and 2001, respectively and the Ph.D. degree from the University of Nottingham, Nottingham, UK., in 2009, all in electrical engineering. In 2002, he was a lecturer in the Electrical and Electronic Department, Rangsit University, Thailand. Since 2003, he has been a Lecturer in the School of Electrical Engineering, SUT. He received the Associate Professor in Electrical Engineering in 2015. His main research interests include system identifications, artificial intelligence applications, stability analysis of power systems with constant power loads, modeling and control of power electronic based systems, and control theory. 